\title{
Robust Estimation of the Apparent Diffusion Coefficient Invariant to Acquisition Noise and Physiological Motion
}

\author{
Santiago Sanz-Estébanez ${ }^{\mathrm{a}, *}$, Tomasz Pieciak ${ }^{\mathrm{a}, \mathrm{b}}$, Carlos Alberola-López ${ }^{\mathrm{a}}$, \\ Santiago Aja-Fernández ${ }^{\mathrm{a}}$ \\ ${ }^{a}$ Laboratorio de Procesado de Imagen, Department of Teoría de la Señal y Comunicaciones \\ e Ingeniería Telemática, ETSIT, Universidad de Valladolid, Campus Miguel Delibes s.n., \\ 47011, Valladolid, Spain. \\ ${ }^{b}$ AGH University of Science and Technology, al. Mickiewicza, 30-059, Kraków, Poland
}

\begin{abstract}
Purpose: In this work we have proposed a methodology for the estimation of the apparent diffusion coefficient in the body from multiple breath hold diffusion weighted images, which is robust to two preeminent confounding factors: noise and motion during acquisition.

Methods: We have extended a method for the joint groupwise multimodal registration and apparent diffusion coefficient estimation, previously proposed by the authors, in order to correct the bias that arises from the non-Gaussianity of the data and the registration procedure.

Results: Results show that the proposed methodology provides a statistically significant improvement both in robustness for displacement fields calculation and in terms of accuracy for the apparent diffusion coefficient estimation as compared with traditional sequential approaches. Reproducibility has also been measured on real data in terms of the distribution of apparent diffusion coefficient differences obtained from different $b$-values subsets.

Conclusions: Our proposal has shown to be able to effectively correct the estimation bias by introducing additional computationally light procedures to the original method, thus providing robust apparent diffusion coefficient maps in the liver and allowing an accurate and reproducible analysis of the tissue.

Keywords: Diffusion Weighted Imaging, Acquisition Noise, Patient Movement Correction, Multimodal Groupwise Registration, Apparent Diffusion Coefficient
\end{abstract}

\footnotetext{
* Corresponding author

Email address: ssanest@lpi.tel.uva.es (Santiago Sanz-Estébanez)

URL: www.lpi.tel.uva.es/ssanest (Santiago Sanz-Estébanez)
} 


\section{Introduction}

Diffusion weighted magnetic resonance imaging (DW-MRI) is a non-contrast technique sensitive to molecular displacement which provides the capability to non-invasively assess microstructure organization within the tissue and cell

5 membrane density upon differences in water proton mobility [1]. DW-MRI is increasingly employed for tissue evaluation as it provides characteristic quantitative parameters of the tissue.

Among the different values that can be derived from the diffusion signal, the apparent diffusion coefficient (ADC) is the most commonly used biomarker, which is obtained after applying a monoexponential decay model to the diffusion images of isotropic tissues [2]. ADC values have shown to be accurate indicators of tumor response to therapy [3,4], as well as the severity of liver fibrosis and cirrhosis [5]. However, in order to thoroughly validate the ADC as an imaging biomarker, a robust parameter estimation methodology suitable

15 for multiparametric acquisitions is necessary. Unfortunately, quantitative ADC mapping is affected by multiple artifacts and confounding factors, including motion-related errors [6], image distortions caused by susceptibility, and noiserelated effects [7]. Although most of the proposed estimation techniques in the literature have focused on the reduction of these artifacts, those methods have usually considered ADC estimation and the reduction of the different confounding factors and artifacts as two different problems that are usually treated separately. Nonetheless, even applying the most complex models and estimation techniques over the diffusion weighted images (DWI), the confounding factors may lead to false conclusions and misestimation of the ADC.

In abdominal organs such as the liver, kidney, or spleen, possible movement of the organ across the different $b$-value scans could result in image misalignment $[8,9]$. Consequently, computing the ADC map on a pixel-by-pixel basis is errorprone, highly likely incurring a boundary smearing [10]. In clinical practice, to reduce this misalignment-related inaccuracies, the region of interest should so be individually segmented in the different $b$-value images to extract the mean signal intensity at this region pixels for the different $b$-values and only then, compute the ADC. However, this methodology does not explicitly account for tissue heterogeneity, thus leading to biased ADC estimates in several clinically relevant scenarios.

35 Breath-hold acquisitions are also a popular way of avoiding respiratory motion artifacts with fast scan times. However, when images with different $b$-values have been acquired at different breath-holds and, subsequently, used for ADC estimation, considerable image artifacts can arise that stem from the fact that two breath hold states are never identical [11].

Extensive research has been carried out on motion-robust sequences in MRI [12]. Most approaches have posed the registration problem from a pairwise standpoint. This procedure, however, is prone to an undesired bias towards the a priori chosen template [13]. On the other hand, groupwise approaches are based on an image reference built out of the whole image set, so the bias 45 mentioned above vanishes. 
Recently, spatially-constrained approaches have also been proposed for liver DWI non-rigid registration (see [14, 15]) grounded on Markov Random Field $(\mathrm{MRF})$, showing an increase in robustness of diffusion parameter estimation by enforcing boundary consistency. Groupwise approaches that use a MRF have

50 also been described [16]. However, the first two approaches are devised for the tracking of small structures and organs, thus requiring a higher degree of regularization. In addition, despite their elegant formulation, computational needs associated to MRF approaches are high. With respect to the latter, in [17], we showed a better performance of our scheme.

${ }_{55} \quad$ In [17], we have previously presented a novel light methodology for joint ADC estimation and groupwise registration of diffusion weighted images in the liver. However, despite its good performance, the method did not take into account any possible bias introduced by the noise present in the data.

The presence of noise in the data is precisely a major issue in DW-MRI, since 60 it greatly affects accurate evaluation of signal decay parameters [18], becoming specially critical at higher $b$-values, where the signal-to-noise-ratio (SNR) decreases. Specifically, it has been shown that insufficient SNR can lead to spuriously low ADC values and a high variance (low precision) in estimation $[19,7]$.

65 The principal source of noise in MR data is the heat source, i.e., the subject or object to be scanned (what is commonly referred to as thermal noise), followed by electronic noise in the receiver chain [20,21], produced by the stochastic motion of free electrons in the coils and by eddy current losses in the patient, which are inductively coupled to the coils. There are different ways to cope with 70 the noise component in the estimation of diffusion parameters; but, due to its random nature, a probabilistic modelling is a proper and powerful solution. The most accepted model is to consider that MR magnitude images are corrupted by Rician noise $[22,21]$. This is a common assumption in MRI, mostly valid for single-coil acquisitions and multi-coil parallel imaging methods reconstructed 75 with a spatial matched filter, like SENSE [23], for instance. In the former, noise depends on a single scalar parameter, $\sigma$ while on the later, noise can become non-stationary, i.e., the variance of noise will depend on the position and $\sigma$ must be replaced by $\sigma(\mathbf{x})$. Nonetheless, many data processing techniques still assume stationary distributions as a model, forgetting about the non-stationarity of

so the data. This is probably due to the fact that most noise estimators in the literature are based on a single noise variance $\left(\sigma^{2}\right)$ value for all the pixels in the image.

It is usually argued that these noise-induced biases do not seriously affect MR images and an identically distributed signal-independent Gaussian model 85 is commonly assumed. However, this may not be suitable when performed over images with relative low SNR, which is the case in DW-MRI in higher b-values [24], since it may lead to the introduction of a noise-induced bias which greatly affect subsequent estimations.

There are in general two ways to cope with noise in estimation: first, to in90 clude the noise model into the estimation pipeline and second, to remove or reduce the noise with some filtering or denoising method. Many authors have pre- 
cisely introduced Rician statistics in the estimation of diffusion models [19, 6, 25] to accurately retrieve ADC estimates, thus significantly increasing computational complexity. On the other hand, denoising techniques [26, 27, 28, 29, 30] have also been developed as preprocessing steps with the purpose of removing the noise-induced bias from the data for the sake of robustifying estimation in subsequent stages.

The purpose of this paper is to define a complete framework that allows the ADC estimation from DW-MRI data taking into account two preeminent con-

100 founding factors: the motion of the patient and the physiological noise present in the data. To that end, the work is grounded on a methodology for joint ADC estimation and groupwise registration that we have previously presented in [17]. Although that scheme has shown to be able to successfully cope with the movement of the patient, it did not take into account any model for the noise. As a consequence, some steps of the procedure may introduce a significant bias. In order to cope with both confounding factors simultaneously, we have incorporated within the original pipeline proper methods which correct for the interpolation bias that arises from the weighting of non-Gaussian (skewed) distributed data, and which, accordingly, improve the ADC estimation. We 110 have shown that the complete algorithm yields higher quality ADC maps when compared to other previously reported approaches.

\section{Theory}

\subsection{Signal and Noise Modelling}

In order to obtain relevant information from a limited amount of acquisitions, it is necessary to assume a diffusion model that allows the estimation of the diffusion measures related to the properties of the different tissues. To that end, many different models have been proposed in the literature [2, 31, 32], where the simplest is the assumption of a monoexponential model to the diffusion weighted signal decay [33]. Such model gives a quantitative diffusion value that 120 has proved to successfully characterize different tissues in the body. This model will be adopted in this paper; nonetheless, the methodology here proposed could be easily extended towards more complex diffusion models.

The acquired complex signal in the monoexponential model is defined as:

$$
A(\mathbf{x})=A_{0}(\mathbf{x}) e^{-b \cdot \operatorname{ADC}(\mathbf{x})},
$$

where $b$ is the weighting of the diffusion sequence, the so-called $b$-value [34], which determines the strength of applied diffusion weighting. $A_{0}(\mathbf{x})$ denotes the complex noiseless signal intensity obtained with a null diffusion gradient. This noise-free DW signal is not available, since the scanner provides a noise corrupted version. For practical purposes, it is usually assumed that the noise in the image domain is a zero-mean, spatially uncorrelated Gaussian process, with equal variance in both the real and imaginary parts [21]:

$$
S(\mathbf{x})=A(\mathbf{x})+N\left(\mathbf{x} ; 0, \sigma^{2}\right)
$$




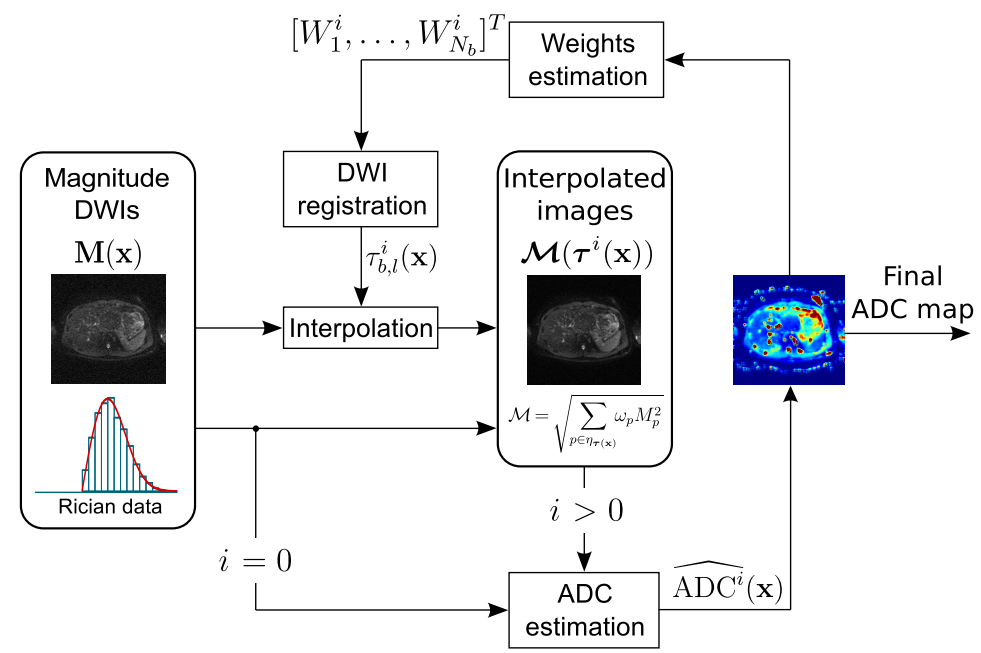

Fig. 1. Pipeline of joint registration and ADC estimation under Gaussian assumptions. Superscript $(i)$ indicates number of iteration, while subscripts $b$ and $l$ indicate $b$-value of the image and displacement direction, respectively.

with $A(\mathbf{x})$ being a noise-free signal and $N\left(\mathbf{x} ; 0, \sigma^{2}\right)$ is a complex Gaussian distributed noise with zero-mean and a constant variance $\sigma^{2}$. In the case that 125 the data is acquired by several receiving coils, the exact same distribution is assumed for all of them.

In clinical and research scenarios, however, it is more usual to work with magnitude data rather than its complex representation. Thereby, in its simplest form, the magnitude information is retrieved by applying the absolute value operator over the complex signal, i.e., $M(\mathbf{x})=|S(\mathbf{x})|$. Hereinafter, we will work with the magnitude images $M$. Since the modulus operator is not a linear transformation, the Gaussianity assumption is no longer valid in magnitude data. Hence, the noise becomes signal-dependent and it is modeled according to Rician statistics [21].

In advanced acquisition techniques that involve parallel imaging, interpolation and other reconstruction techniques, different noise models may arise. However, many of them may yield to non-stationary Rician distributions: the noise variance $\sigma^{2}(\mathbf{x})$ is no longer constant, but it depends on the position $\mathbf{x}$ within the image. Hereinafter, we will consider both variants, i.e., stationary and non-stationary Rician distributed magnitude DW data. The method here proposed can be easily extended to other noise models, such as the nc- $\chi$ distribution.

\subsection{Motion Correction and $A D C$ estimation}

To overcome the issues related with the misalignment between the different $b$-value images, we must accurately retrieve the motion compensating displacement fields, so that we can obtain a consistent representation of the organ of 
study, thus avoiding blurred ADC estimates and motion artifacts. Due to the significant signal intensity dropout observed in the images when increasing the $b$-value, it is mandatory to resort to multimodal methodologies.

Therefore, we have made use of the method we have previously proposed in [17], a methodology to jointly estimate the ADC map and register DWMRI sequences acquired in different breath hold states. The processing pipeline has been depicted in Fig. 1. Briefly stated, the procedure performs an elastic multimodal groupwise registration step in which the transformation $\boldsymbol{\tau}$ (see Eq. (4)) has been defined as a combination of B-spline free form deformations (FFDs) [35]. The objective function to be optimized for the registration problem presents the function $H$ in Eq. (4) as data fidelity term; this function is based on Eq. (1) as the forward model, which should match the measured DW magnitude data as defined in [17]:

$$
\left.H(\mathbf{M}, \mathrm{ADC}, \boldsymbol{\tau})=\sum_{b=1}^{N_{b}} W_{b}^{i}\left(\mathcal{M}_{b}\left(\tau^{i}(\mathbf{x})\right)\right)-\widehat{M}_{b}\left(\operatorname{ADC}^{i}(\mathbf{x})\right)\right)^{2},
$$

where $\widehat{M}_{j}$ is the estimated intensity value (in magnitude) from the current (ith) ADC estimate obtained by applying Eq. (1) for a given $b$-value $b$ out of a total of $N_{b}$. The proposed metric additionally incorporates a $N_{b}$-component vector $\mathbf{W}$ which weighs each component of the data fidelity term according to the expected signal content, i.e., weights are a decreasing function of the ${ }_{55} b$-value [36]. This weighting vector is initially constant and unitary but their components are updated along the iterations. The transformation $\tau$ has been driven to be smooth by means of a penalty term based on its first and second order derivatives, resulting in the following optimization problem:

$$
\begin{array}{r}
\boldsymbol{\tau}^{i}=\underset{\boldsymbol{\tau}}{\operatorname{argmin}} \int_{\chi}\left(H\left(\mathbf{M}, \widehat{\mathrm{ADC}^{i}}, \boldsymbol{\tau}\right)+\sum_{b=1}^{N_{b}} \sum_{l=1}^{L} \lambda_{1}\left(\sum_{l^{\prime}=1}^{L}\left(\frac{\partial \tau_{b, l}(\mathbf{x})}{\partial x_{l^{\prime}}}\right)^{2}\right)+\right. \\
\left.\lambda_{2}\left(\sum_{l^{\prime}=1}^{L} \sum_{l^{\prime \prime}=1}^{L}\left(\frac{\partial^{2} \tau_{b, l}(\mathbf{x})}{\partial x_{l^{\prime}} \partial x_{l^{\prime \prime}}}\right)^{2}\right)\right) d \mathbf{x},
\end{array}
$$

where $\mathbf{M}$ are the magnitude images and $\tau_{b, l}$ represents each of the displacement components of the transformation (with $L=2$ for the $2 \mathrm{D}$ case) for each $b$-value image. Derivatives have been approximated by finite differences in the spatial dimension $\mathbf{x}$. The influence of the regularization term has been balanced by $\lambda_{1}$ and $\lambda_{2}$. Quadratic interpolation has been used to obtain the intensity of the deformed images on a rectilinear grid.

For the ADC estimation, the optimization functional comprises the same weighted metric $H$ as used for the registration procedure and an additional Total Variation (TV) regularization term on the ADC map to ensure smooth estimates. Specifically:

$$
\widehat{\mathrm{ADC}^{i}}=\underset{\mathrm{ADC}}{\operatorname{argmin}} \int_{\chi}\left[H\left(\mathbf{M}, \mathrm{ADC}, \boldsymbol{\tau}^{i-1}\right)+\beta\|\mathrm{ADC}(\mathbf{x})\|_{\mathrm{TV}}\right] d \mathbf{x},
$$


with $\|\mathrm{ADC}(\mathbf{x})\|_{\mathrm{TV}}$ the spatial TV on the ADC which is controlled by a nonnegative constant $\beta$. We should stress that the parameters involved in the estimation problem, particularly the ones related to the spatial TV term in Eq. (5), may greatly influence the performance of this step. A fine tuning of these parameters is needed in order to prevent the TV term from oversmoothing the structure in the ADC map so that its impact is just a mild denoising. However, notice that, by placing the TV ADC estimation at the end of the pipeline, it will not interfere with other stages of the procedure, thus keeping magnitude images untouched and simplifying parameter setting.

In this original formulation, no noise model has been taken into account.

175 That could not be a problem in Gaussian distributed data, but in distributions with certain skewness (like Rician, Rayleigh, nc- $\chi$ or Gamma), operations like those carried out in Fig. 1 are known to produce certain bias that can seriously affect the output values of the different steps (specially due to the iterative nature of the procedure) and consequently the accuracy of the final ADC estimate. In the following section, those biases introduced by the presence of noise, will be analysed and properly corrected.

\subsection{Noise-induced Bias Correction}

According to the scheme described in Fig. 1, the ADC has been estimated from the registered magnitude images, which have been obtained through interpolation using the transformation $\tau$ previously estimated. The interpolated (squared) images $\mathcal{M}^{2}(\boldsymbol{\tau}(\mathbf{x}))$ have been obtained as:

$$
\mathcal{M}^{2}(\boldsymbol{\tau}(\mathbf{x}))=\sum_{\mathbf{x}_{p} \in \eta_{\boldsymbol{\tau}(\mathbf{x})}} \omega_{p} M^{2}\left(\mathbf{x}_{p}\right),
$$

with $\eta_{\boldsymbol{\tau}(\mathbf{x})}$ a square neighborhood around the transformed pixel. If the original magnitude data $M(\mathbf{x})$ are assumed to follow a Rician distribution, data after a rigid registration will still remain Rician distributed, since no operation are done on the values of the different pixels, only on the positions. Thus, the squared interpolation can be seen as the weighted sum of squared Rician signals. We have calculated the expected value of the output (for the sake of simplicity, we drop spatial dependence and denote transformed pixel as $j$ with neighborhood $\left.\eta_{j}\right)$ :

$$
\begin{aligned}
\mathbb{E}\left\{\mathcal{M}_{j}^{2}\right\} & =\mathbb{E}\left\{\sum_{p \in \eta_{j}} \omega_{p} M_{p}^{2}\right\}=\sum_{p \in \eta_{j}} \omega_{p} \mathbb{E}\left\{M_{p}^{2}\right\} \\
& =\underbrace{\sum_{p \in \eta_{j}} \omega_{p} A_{p}^{2}}_{\text {original interpolation }}+\underbrace{2 \sum_{p \in \eta_{j}} \omega_{p} \sigma_{p}^{2}}_{\text {bias }} .
\end{aligned}
$$

It can be seen that the last term corresponds to a bias, which should not be neglected in low SNR scenarios. Besides, this bias will be fed back inside the optimization loop, thus increasingly affecting ADC estimates. 


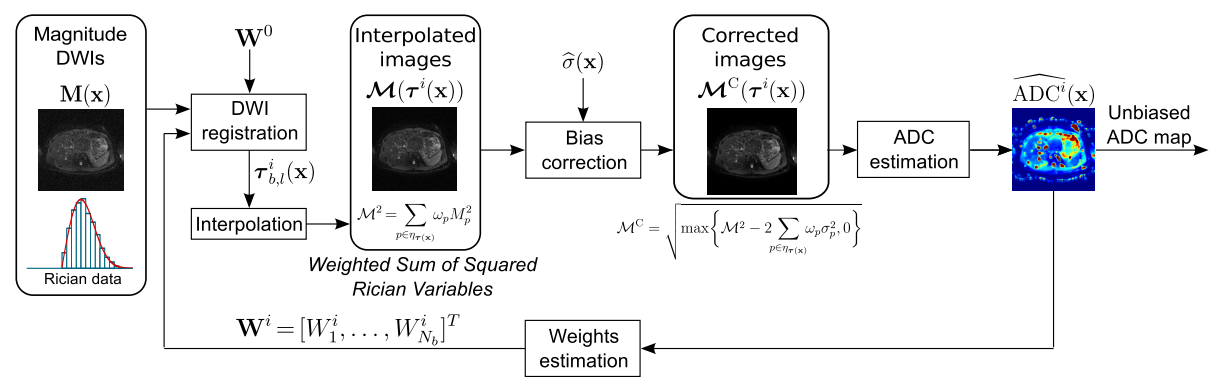

Fig. 2. ADC estimation procedure with motion compensation and noise-induced bias correction. $\mathbf{W}^{0}$ is the initial weighting parameter and $\widehat{\sigma}(\mathbf{x})$ is an estimate of the noise variance.

In order to remove this bias in Eq. (7), we have decided to avoid any filtering of the data. Although filtering techniques have shown proper performance in many estimation problems, we have preferred to keep the original data whenever possible, to avoid any loss of information that overfiltering may produce. In this case, we can easily remove this noise-induced bias by estimating the signal $\sum_{p \in \eta_{j}} \omega_{p} A_{p}^{2}$. If we know the value of the variance of noise for each pixel in the image $\sigma_{p}^{2}$, we can simply define the interpolated signal as:

$$
\left(\mathcal{M}_{j}^{\mathrm{C}}\right)^{2}=\mathcal{M}_{j}^{2}-2 \sum_{p \in \eta_{j}} \omega_{p} \sigma_{p}^{2}
$$

The non-squared signal will be finally obtained as:

$$
\mathcal{M}_{j}^{\mathrm{C}}=\sqrt{\max \left\{\sum_{p \in \eta_{j}} \omega_{p} M_{p}^{2}-2 \sum_{p \in \eta_{j}} \omega_{p} \sigma_{p}^{2}, 0\right\}} .
$$

The maximum operator has been introduced to avoid any negative term inside the square root. If noise is assumed to be stationary, the variance of noise is the same for all the image and therefore the parameter $\sigma_{p}^{2}$ simply becomes $\sigma^{2}$.

The ADC estimation pipeline with the inclusion of the interpolation bias correction is shown in Fig. 2, where $\mathbf{W}^{0}$ represents the initial weighting parameter and $\widehat{\sigma}^{2}(\mathbf{x})$ is an estimate of the noise variance. For the sake of generalization, we have assumed the latter to be spatially dependent.

It is worth pointing out that the suitability of the Rician bias correction procedure will greatly depend on the local SNR; when a high SNR is present, 195 a Gaussian distribution can be assumed, so no bias should appear in the data. On the other hand, when the signal tends to zero, data becomes Rayleigh distributed, so Rician correction may be inaccurate. Therefore, our method will have more clear benefits on tissues with shorter T2; overall, any acquisition parameter that affects either the signal content (TE, mono or multi-echo se200 quence, b-value) of the noise (receiver bandwidth) will have an impact in the model suitability. 


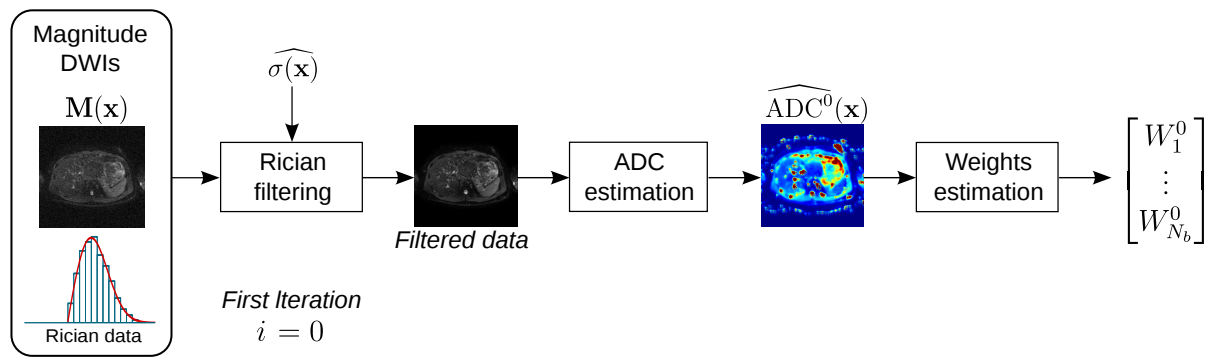

Fig. 3. First iteration of the ADC estimation procedure: a Rician noise filtering step is introduced prior to the first estimation of the $\mathrm{ADC}$ and the registration weights.

In order to obtain the initial weighting parameter $\mathbf{W}^{0}$, a previous ADC estimation has to be performed. The original Rician data is used for this estimation, since no prior registration is carried out. As a consequence, the noisy data are 205 directly used in this estimation. In order to reduce the bias and variance of this previous step, we propose to introduce a Rician noise filter. The filtering will only be used in this first step. Once the first set of weights is available, this filter is no longer needed, since the bias can be directly removed without any denoising step. That way, we avoid any possible overfiltering of the images that may distort anatomical details and hinder the subsequent registration. For clarity, Fig. 3 sketches the detailed processing in the initial iteration.

One major issue to be also considered in the proposed pipeline is a robust noise estimation. Our method requires the noise variance $\sigma^{2}$ to be known or at least (smoothly) estimated from the data both from the background and the foreground regions. Methodologies widely employed to estimate stationary $\sigma^{2}$ can be found in [21].

For the non-stationary Rician case, a more complex noise estimation step is needed due to the spatially variant nature of the noise variance $\sigma^{2}(\mathbf{x})$. Therefore, we have resorted to a method proposed in [37, 38], that uses a variance220 stabilizing transformation which transforms the magnitude data from a signaldependent noise to an independent one. Afterwards, the spatial variability of noise has been retrieved by a homomorphic filtering. We have restricted the spatially variant noise estimators to operate only over a predefined region avoiding outer organs influence. This procedure is able to estimate the spatially variant noise maps using only a single image at fixed $b$-value, which is the case considered here. Noise parameters have been precalculated and then stored for its later use inside the algorithm loop.

Finally, for the sake of completeness, we show in Fig. 4 the whole processing pipeline in which the two-tier processing is clearly depicted. 


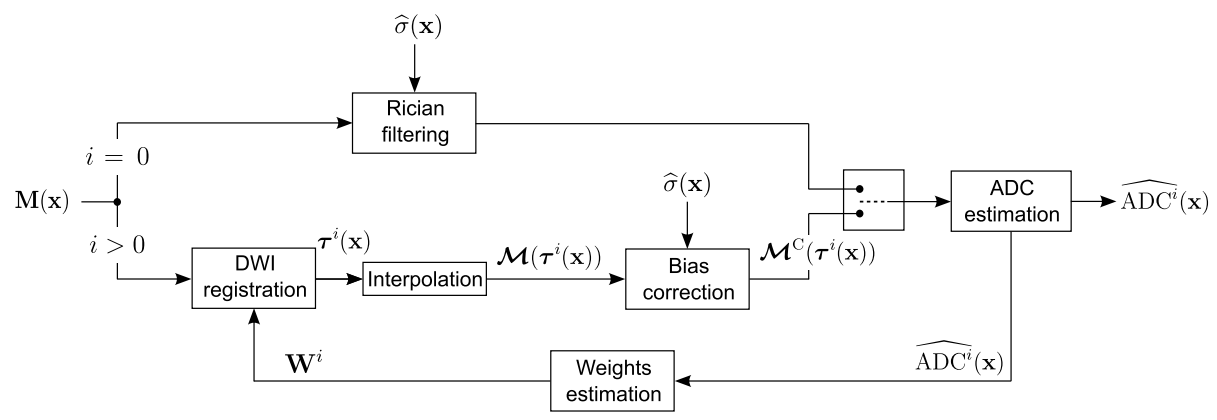

Fig. 4. Pipeline of the proposed ADC estimation method. The method corrects the motion of the patient through registration while reduce the bias induced by Rician noise. The switch drawing indicates that ADC estimation takes as input images the ones coming from the upper branch only at first iteration whereas taking them from the lower branch in subsequent iterations.

\section{Materials and Methods}

\subsection{Materials}

\subsubsection{Diffusion Simulation}

DW-MRI synthesis has been subdivided in two parts. Firstly, the $b_{0}$ image has been simulated; in this work we made use of the well-known Spin Echo sequence, for the intensity values in the image are given by [39]:

$$
A_{0} \propto \rho\left(1-e^{-T_{R} / T_{1}}\right) e^{-T_{E} / T_{2}},
$$

where $\rho, T_{1}, T_{2}$ stand for the proton density, longitudinal and transverse relaxation times assigned for each simulated tissue in the volume, respectively.

Secondly, we have simulated isotropic diffusion by a random walk process using the Monte-Carlo method according to the methodology presented in [40]. Particles were uniformly distributed in the voxel and the trajectory of a spin was generated by randomly moving the particle during each walk. The final diffusion signal, derived from the bulk phase shift $\Phi_{i}^{k}$ of the $K$ simulated particles, can be numerically approximated $[41,42]$ by:

$$
A=\frac{A_{0}}{K} \sqrt{\left(\sum_{i=1}^{K} \cos \left(\Phi_{i}^{k}\right)\right)^{2}+\left(\sum_{i=1}^{K} \sin \left(\Phi_{i}^{k}\right)\right)^{2}},
$$

where $A_{0}$ is the noiseless $b_{0}$ image intensity from Eq. (10). Complex Gaussian noise has been added afterwards according to Eq. (2). We have simulated the following $b$-values: $b \in\{0,10,20,50,100,200,500,1000,1500,2000\} \mathrm{s} / \mathrm{mm}^{2}$. Sampling of $b$-values is finer for lower $b$-values according to [43].

\subsubsection{Synthetic Data}

For validation, we have developed a synthetic diffusion phantom using a simulation environment based on the 4D extended cardio-torso (XCAT) phantom 
[44]. The phantom is built from a whole body model that contains high level detailed anatomical labels, which feed a high resolution image synthesis procedure. The 4D XCAT phantom incorporates state-of-the-art respiratory and cardiac mechanics, which provide sufficient flexibility to simulate non-rigid motion effects on the liver from a user-defined parameter set. Therefore, the phantom will provide us not only with the images themselves, but also with ground-truth deformation fields and a synthetic reference ADC map. For the ADC simulation, we have covered the whole volume of the liver (both lobes). The parameters of 250 the bulk phase shift simulation have been set so that the ground-truth simulated ADC values present a distribution with $1.6 \pm 0.4 \times 10^{-3} \mathrm{~mm}^{2} / \mathrm{s}$ (mean $\pm \mathrm{std}$ ).

The resulting simulated images are shown in Fig. 5, from which the global influence of the different breath holds on each image can be observed, as well as the simulated decreasing SNR (Rician stationary noise). Moreover, SENSE 255 acquisitions have also been simulated. Therefore, non-stationary noise has been generated, which presents a spatial pattern that depends on a number of imaging parameters [21] such as, coils sensitivities, SENSE acceleration factor and correlation between coils. An exemplary synthetic non-stationary noise pattern is presented in Fig. 5.
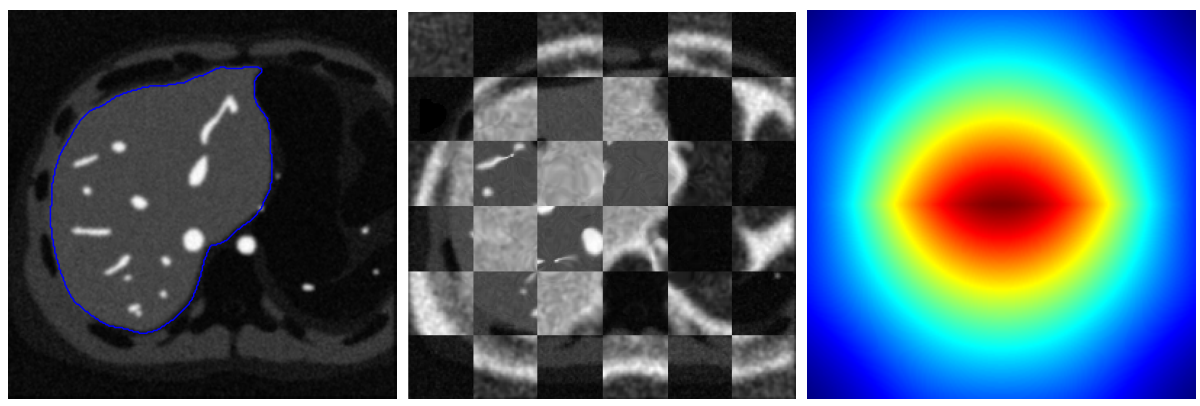

Fig. 5. Axial slices of the simulated diffusion sequence. The figure on the left shows the image corresponding to the $b_{0}$, as well as the ROI $(\chi)$ boundary in blue, while the figure on the center is a checkerboard with $b$-values of 100 and $1000 \mathrm{~s} / \mathrm{mm}^{2}$. The figure on the right shows noise map for non-stationary Rician noise simulation with an acceleration factor of 2 and correlations between coils set to 0.1 .

\subsubsection{Real Data}

Additionally, we have performed MRI acquisitions over a sample of four healthy volunteers. Axial SENSE DWI and T2 weighted Turbo Spin Echo sequences have been acquired on a Philips Achieva 3T scanner in each case of study. The latter will be used to manually delineate the whole liver as region of 265 interest (ROI) $\chi$ on which meaningful measurements will be obtained. All the subjects signed the ordinary informed consent for the MR session and agreed in writing to share the resulting images for research purposes. Personal data were treated according to current legislation. Sequence details for real and synthetic data are shown in Table 1.

Ten $b$-values have been chosen to be common for every sequence and volun- 
Table 1

Details on the image sequences used in the paper. $\Delta_{p}$ : Spatial Resolution $(\mathrm{mm}) . \Delta_{l}$ : Slice Thickness $(\mathrm{mm}) . N_{p}$ : Number of pixels along each direction. $N_{b}$ : Number of b-values. $N_{s}$ : Number of slices. $T_{E}$ : Echo Time (ms). $N_{g}$ : Number of diffusion gradients. Card.: Cardiac Period (s). Resp.: Respiratory Period (s).

\begin{tabular}{rrr}
\hline Params. & XCAT & DWI volunt. \\
\hline$\Delta_{p}$ & 1 & $1.23-1.85$ \\
$\Delta_{l}$ & 10 & 5.5 \\
$N_{p}$ & 268 & $224-320$ \\
$N_{b}$ & 10 & 10 \\
$N_{s}$ & 21 & $20-40$ \\
$T_{E}$ & 93 & $65.65-72.1$ \\
$N_{g}$ & 1 & 3 \\
Card. & 1 & $\sim 1$ \\
Resp. & 4 & Free \\
\hline
\end{tabular}

teer.

\subsection{Methods}

\subsubsection{Reference Methods}

Both real and synthetic datasets have been processed using different groupwise multimodal metrics [45] as well as pairwise registration methodologies. As for the former, apart from the pipeline here proposed, we have tried the Entropy of the distribution of intensities (Entr.) [46], Modality Independent Neighbourhood Descriptor (MIND) [47] and the joint formulation proposed in [17] based on weighted ADC residuals (bWei).

As for the latter, the Demons [48] registration algorithm (DEM) has been implemented using a mutual information based metric; this registration algorithm has been implemented under the pairwise paradigm. Therefore $b_{0}$ image has been selected as the image template.

These methodologies have been built as sequential approaches (except for the joint procedure) as they focus on registration only. Consequently, ADC estimates are obtained once the DWI dataset is fully registered. We have solved the Non-Linear Least Squares problem for ADC estimation using the LevenbergMarquardt method.

On the other hand, for the Rician noise filter we have implemented different 290 literature filtering approaches to compare its performance inside our proposed pipeline. Namely, we have tested a reported linear minimum mean square error (LMMSE) procedure [26, 27], a Wiener filter (assuming a Gaussian noise model), an unbiased non-local means (UNLM) denoising scheme $[49,50]$ and a non local transform-domain filter (BM4D) [51].

\subsubsection{Evaluation on synthetic data}

We have first carried out a comparison using the data provided by the XCAT computational phantom for the different filtering procedures described in Section 3.2.1 under different scenarios in order to assess its suitability. We have also measured the improvement due to the bias correction step, i.e., the pipeline 
$[17]$ is also shown for reference.

Furthermore, for the synthetic experiment we have simulated different types of noise both stationary ("St.") and non-stationary ("NS") Rician noise, the latter using the noise map represented in Fig. 5. Hence, we can measure both 305 the error in the estimation of the simulated deformation field $\boldsymbol{\tau}$ and accuracy in ADC estimation for the methodologies presented in Section 3.2.1.

Besides, we have compared our proposal with respect to the approach described in [17], in which the joint estimation-registration is performed although under an underlying Gaussian assumption in order to assess robustness towards the ADC map estimated for stationary and non-stationary noise with different noise variances.

We have always used the ground-truth noise variance $\sigma^{2}(\mathbf{x})$ in the synthetic experiments so as to avoid possible biases from noise estimation methodologies. Experiments have been performed 100 times with different noise executions.

\subsubsection{Evaluation on real data}

For the real data, due to the absence of a ground-truth, validation has to be performed by indirect measures and qualitative visual assessment. We, therefore, have proposed an indirect validation procedure based on the esti-

320 mates of the ADC obtained with different subgroups of b-values, with lengths of $n_{b}=[3,6,8]$, out of the total number of $b$-values available $\left(N_{b}=10\right)$. Specifically, we have performed $K_{b}=\left(\begin{array}{l}N_{b} \\ n_{b}\end{array}\right)$ ADC estimations for each pixel per slice and patient, changing only the combination of the b-values (maintaining the same number of $b$-values) that enter the optimization problem. Ideally, for 325 two combinations of $n_{b}$ values, the ADC estimates should be pixelwise equal. Therefore, a natural measure of the method reproducibility is the similarity between all ADC estimates. Accordingly, we have calculated the absolute differences between each possible pair out of the $K_{b}$ ADC estimates, giving rise to $K_{b}\left(K_{b}-1\right) / 2$ possible comparisons for each pixel in the liver, which will conform the distribution of ADC differences. Robustness will be measured in terms of the variability of this pattern, the more variable, the less robust the method.

The value of $\sigma(\mathbf{x})$, needed as an input parameter, has been estimated from the data using the method in [37] over a predefined region of interest (ROI) avoiding the influence of outer organs.

\section{Results}

\subsection{Results on Synthetic Data}

Fig. 6 shows boxplot diagrams of the error distributions in ADC estimation for different filtering procedures (as enumerated in Section 2.3) with respect to the original bWei approach. We have also included the results without filtering. 340 From the results, we can conclude that the bias correction step itself is beneficial in order to improve ADC estimation. Regarding noise reduction at the first 

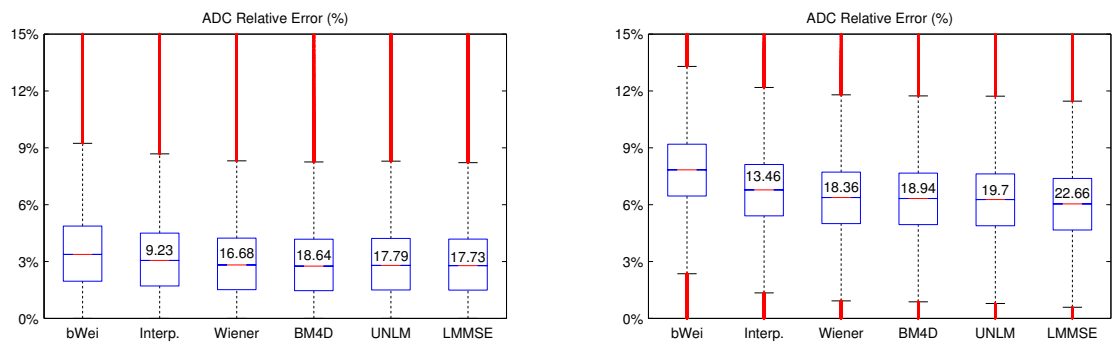

Fig. 6. Relative error on ADC estimates for the different customized filtering schemes described in Section 3.2.2 for stationary noise $(\sigma=[10,35]$, in left and right figures, respectively). Numbers inside the boxes indicate the error reduction (\%) with respect to original pipeline.

iteration, it is observed that LMMSE filter outperforms other state-of-the-art approaches; the gain is observed not only in precision, as shown in the figure, but also in speed.

Mann-Whitney U-tests were performed for the ADC error distributions on both scenarios. Significant differences were found for each of the different filtering procedures with respect to the non-filtered scheme (greater differences were found with respect to the original bWei approach). Best figures were obtained with the LMMSE filter, $p=0.0031$ and $p<10^{-3}$ for scenarios in Fig. 6, with $\sigma=10$ and $\sigma=35$, respectively ( $p<10^{-6}$ against bWei for both). Therefore, hereinafter, this filtering method will be used.

We now test the ability of the proposed methodology on motion correction and robust ADC estimation. We have measured the relative error (over a predefined ROI $\chi$ ) on the ADC estimates under the noise scenarios described in Section 3.2.2 for the different methodologies enumerated in Section 3.2.1 as well as our proposal.

In Fig. 7 we show the boxplot diagrams of the relative error distributions from ADC estimation as a measure of estimation accuracy. Besides, we have also measured the error module of the estimated deformation fields with respect to the ground-truth provided by the XCAT phantom.

Mann-Whitney U-tests have also been performed for the ADC error distributions obtained with our method and with the others in the same scenario. Significant differences were found, although greater in the non-stationary scenario $\left(p<10^{-3}\right)$ than in the stationary case $(p<0.05)$ for all methods. To diminish correlation effect in these tests, images have been decimated by a factor of ten, i.e., pixels entering the tests are taken 10 pixels apart at the acquired resolution.

From results in Fig. 7, we can state that the proposed methodology is effective at improving robustness when obtaining ADC estimates from DW370 MRI sequences and, besides, provides accurate deformation measures, specially when compared to other sequential state-of-the-art techniques, in stationary and spatially-variant noise scenarios; results for the second case are, as expected, worse. Fig. 8 shows the evolution of the ADC estimation error with respect 

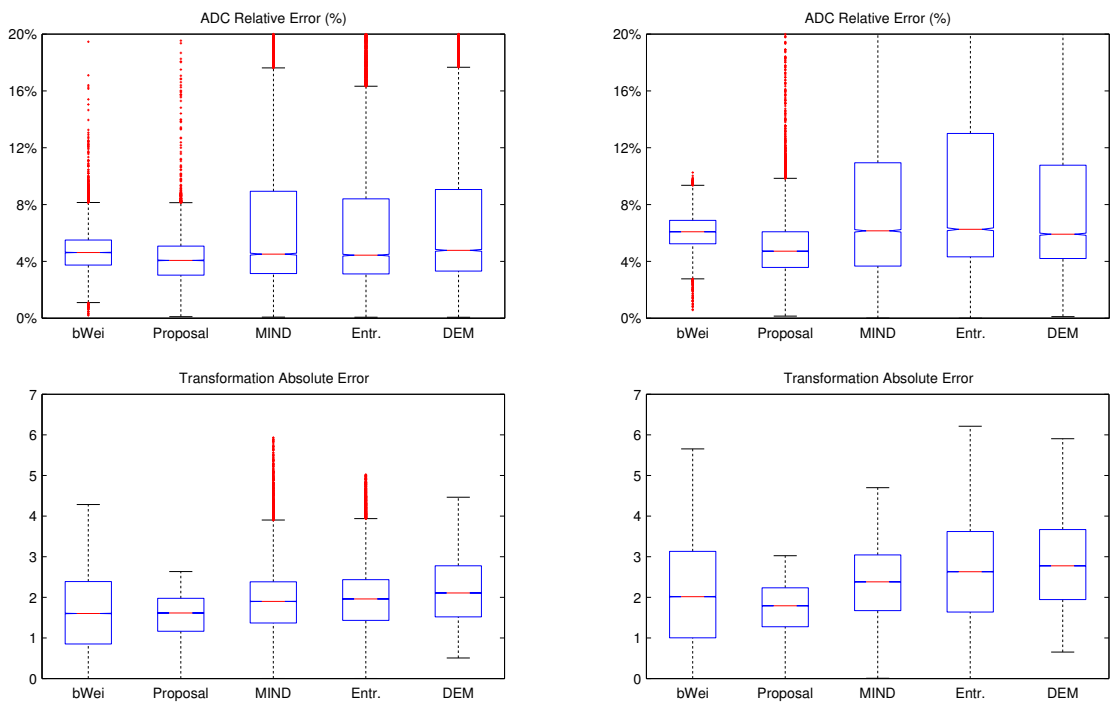

Fig. 7. Relative error on ADC estimation (first row) and module error (mm) for the deformation field (second row) obtained with the different methods in stationary $(\sigma=20)$ and non-stationary (with $\sigma(\mathbf{x})$ median set to 20 ) noise contaminated scenarios, shown in the left and right respectively.

to the noise level (both stationary and spatially-variant) for the original joint registration-estimation approach [17] and our present proposal.
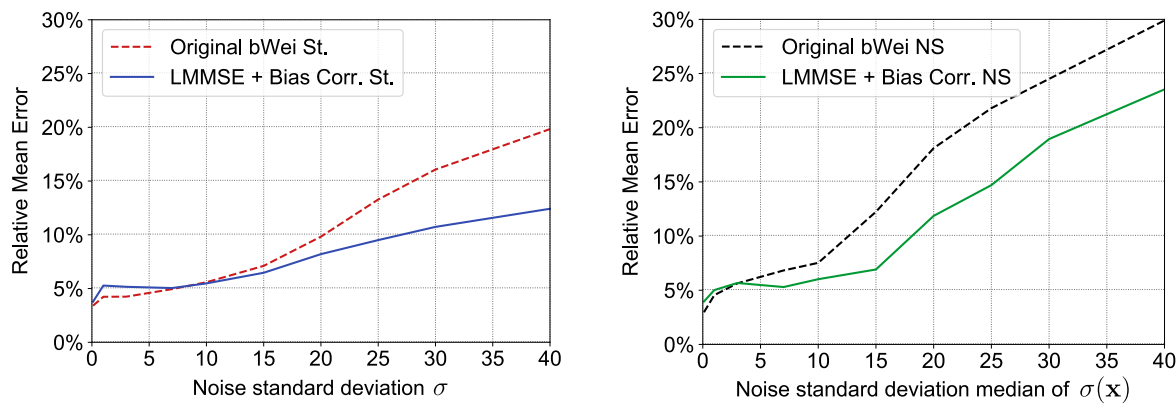

Fig. 8. Mean relative error (\%) on ADC estimates in presence of different noise levels $\sigma$ for both approaches. In the non-stationary noise scenario the x-axis shows the median of $\sigma(\mathbf{x})$.

375

We can see from Fig. 8 that the here proposed methodology can provide accurate estimation under very noisy conditions, with mean error being below $10 \%$ until $\sigma=25$ and $\sigma=15$ for the stationary and non-stationary cases, respectively. This is specially relevant when performance is compared with the original 380 bWei approach, which exponentially decreases for $\sigma>15$. The figure also indicates that at higher SNR differences in estimation between traditional Gaussian procedure and our Rician-based proposal can be neglected, as expected. 


\subsection{Results on Real Data}

Evaluation on real data has been performed according to the scheme described in Section 3.2.3. As sketched in Fig. 9, we have used the cumulative distribution (CD) of the absolute differences on the ADC estimates obtained so as to show the reproducibility of the different methodologies for different number of acquired b-values. Proposal without TV regularization has also been plotted so one can assess the influence of TV step over ADC estimates for the different b-values subgroups.
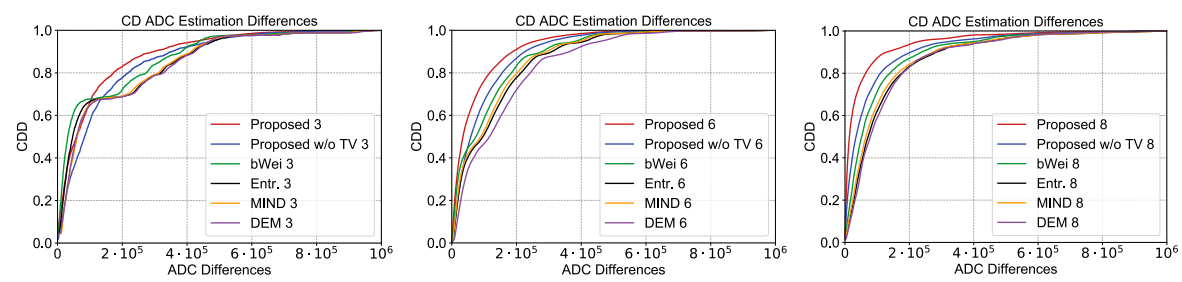

Fig. 9. Cumulative distribution of absolute differences on ADC estimates for the different methods with a fixed number of $b$-values: $n_{b}=3$ (left), $n_{b}=6$ (center) and $n_{b}=8$ (right).

It can be observed in Fig. 9 that the proposed method shows the highest figures, specially when a lower number of b-values is employed and, besides, the curves show a more "monotonic" behavior, what constitutes a good trend towards the absence of bias in the ADC estimation, both in the boundaries and in the interior of the liver. Nevertheless, notice that, since we are resorting to reproducibility measures in the real data, bias in ADC estimation cannot be represented. Therefore, possible errors in the estimation, either caused by the noise variance estimation method or by the monoexponential model assumption, which does not account for the perfusion component in the data, will not be reflected.

Finally, in Fig. 10 we show some snapshots on the resulting estimated ADC to visually notice the noise removal ability of the proposed methodology over real data. From visual inspection, we can support that our approach effectively removes the noise in final ADC maps maintaining liver structures integrity. Fig. 10 also shows a reduction in the smearing near tissue boundaries, which will allow delineation of structures inside the liver directly over the reconstructed ADC map.

\section{Discussion}

The ADC is known to provide useful information in vivo about body tissues, specially in abdominal organs. However, the accuracy of the estimation of this parameter is affected by different external factors, some related to the estimation procedure itself, some to the accuracy of the adopted model and some to the so-called confounding factors. In this work, we have focused on the latter: the reduction of the influence of two preeminent confounding factors in order 

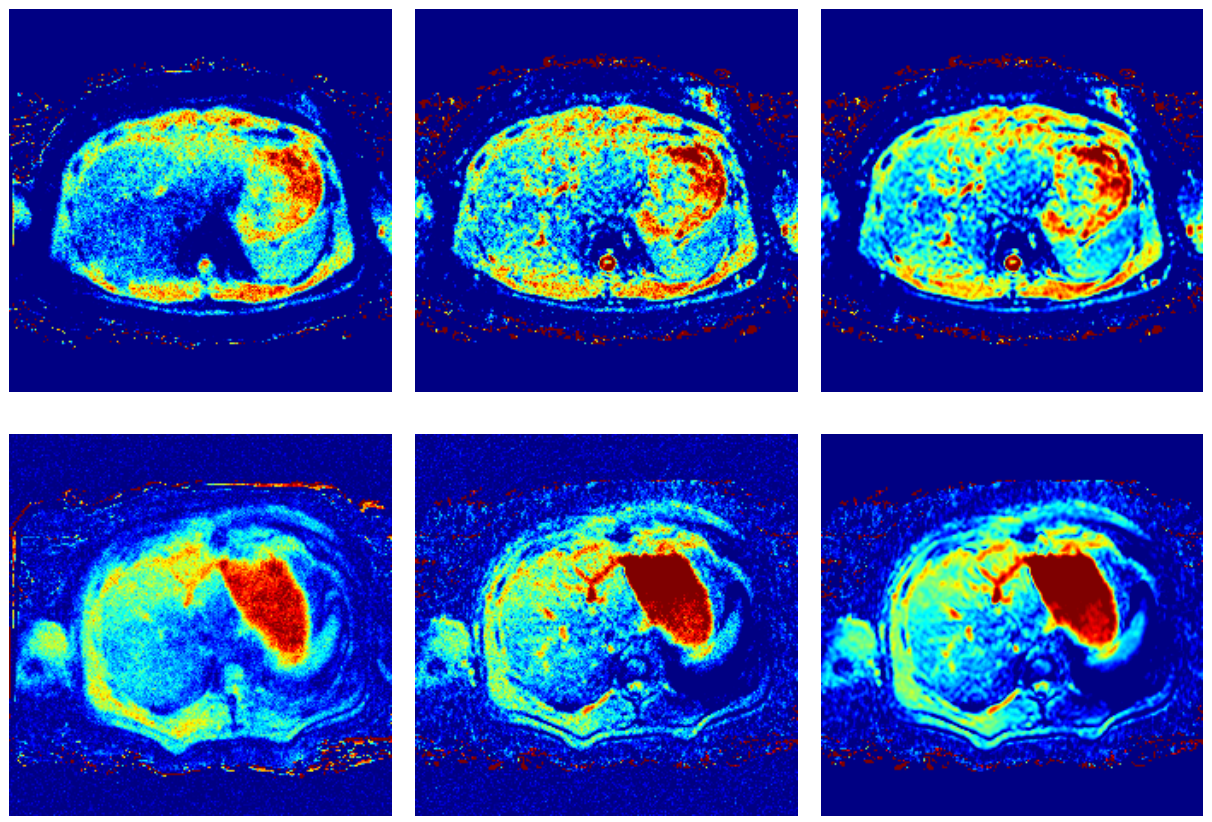

Fig. 10. Real ADC maps for two different patients estimated directly from the original acquired sequence (left) as well as when using the bWei approach (center) and the here proposed methodology with bias correction (right).

415 to improve the accuracy in the estimation of the ADC. To that end, we have presented a step-by-step approach for simultaneous correction of motion artifacts and removal of noise-induced bias in the ADC estimation from DW-MRI sequences acquired in different breath hold states.

The proposed methodology has shown to be specially accurate in recovering ADC maps, showing a significant reduction of the estimation bias when compared to previous approaches. The bias correction procedure plays an important role, improving the performance, specially when the Gaussian assumption does not hold, as shown in Fig. 8. It is important to point out that the whole scheme is grounded on the assumption of an underlying noise model for the acquisi425 tions. In this work we have chosen the Rician distribution, for being the most accepted model in MRI. However, other models can also be easily adopted, without many changes in the general scheme. The relevant issue is precisely to adopt a model: overlooking the use of a statistical model for signal and noise may lead to implicitly assume non-skewed distributions.

As a design choice we have opted to avoid using any filtering procedure to reduce noise. Instead, we have removed the interpolation bias of the data by a simple subtraction. One may discuss that, this way, we only reduce the bias of the estimation, but not the variance. While this is true, we have taken a conservative approach: we believe that it is more important to avoid any obliteration of the original data by overfiltering than to reduce the estimation 
variance.

As a matter of fact, a filter was indeed used, but only on the first iteration in order to obtain an initial set of weights. We have tested different methods and we have finally resorted to LMMSE filtering as preprocessing step since it provides the best performance as shown by Fig. 6 but also due to its lower computational complexity. From Fig. 6, we can also state the relevance of initial iterations in the procedure since the introduction of a filtering stage has significantly improved performance not only with respect to the original proposal (bWei) but also when the first ADC estimation is directly performed 445 over magnitude images.

The proposed pipeline has also proven to be robust towards different types of noise, both stationary and spatially-variant. It has been shown that just by introducing a computationally light stage after interpolation we were able to correct the bias stemming from data weighting without apparently increasing estimates variance, even for low SNR datasets (i.e., variance effects of estimating unknown quantities in Eq. (9) do not seem relevant). Hence, the introduction of more complex models in the optimization equations may slightly improve performance but at the price of greatly increasing computational complexity of the algorithm.

${ }_{455}$ With regards to the estimation of the deformation fields, we can see in Fig. 7 that the mean absolute error seem has experienced only a slight improvement as compared to the original proposal (bWei); however, robustness has been significantly increased. This robustness gain is mainly caused by improvement of the registration performance nearby tissue interfaces inside the liver due to bias removal. This bias reduction (coming both from the groupwise registration and Rician correction) allowed a better alignment with the ADC reference, specially at higher b-values on which these boundaries are barely distinguishable. For this reason, smearing in the ADC maps obtained from the motion compensated DW-MRI sequences has been greatly reduced, which in turn will also benefit 465 the subsequent registration stage.

Notice that both procedures, noise bias correction and motion compensation, have shown its benefits towards robust ADC estimation separately. However, in this paper we have also demonstrated that its simultaneous use is beneficial for each other when employed inside the iterative procedure, providing addi470 tional values with respect to sequential approaches or breath-hold acquisitions. Furthermore, the unified framework has been validated with in vivo human data, suffering from involuntary motion using reproducibility figures. From the improvement observed in Fig. 9, we can see that our procedure allows higher flexibility towards the chosen $b$-values as well as to the total number of images 475 acquired. Furthermore, we can observe that the introduced TV term seems beneficial in preventing noise, although a fine tuning is necessary to avoid oversmoothing in ADC estimates. Visual assessment from Fig. 10 also reveals the motion compensating capability of the method, producing detailed ADC maps, specially as compared to the ones obtained from the original scanner sequence.

${ }_{480}$ Therefore, we can support that the proposed pipeline is suitable to be embedded into direct $\mathrm{ADC}$ reconstruction acquisition protocols, which will not only 
reduce scan time but also possible variabilities due to $b$-value selection.

\section{Conclusions}

In this paper we have extended the joint registration and ADC estimation procedure for DW-MRI sequences in order to provide unbiased ADC estimates. To this end, we have designed a processing pipeline with smartly located filtering stages capable of dealing with the possible biases generated inside the optimization loop.

Experimental results demonstrate that the proposed approach helps to ro490 bustly cope with non-rigid motion artifacts, thus providing an accurate estimation under noisy (also for non-stationary) environments showing higher robustness and reproducibility towards low SNR datasets and b-value selection.

\section{Acknowledgments}

This work was partially supported by the European Regional Development Fund (ERDF-FEDER) under Research Grants TEC2014-57428-R and TEC201782408-R and the Spanish Junta de Castilla y León under Grant VA069U16. Tomasz Pieciak acknowledges National Science Centre (2015/19/N/ST7/01204).

\section{References}

[1] Jones D. Diffusion MRI. Oxford University Press; 2010.

[2] Le Bihan D, Breton E, Lallemand D, Grenier P, Cabanis E, Laval-Jeantet M. MR imaging of intravoxel incoherent motions: Application to diffusion and perfusion in neurologic disorders. Radiology 1986;161(2):401-407.

[3] Kim T, Murakami T, Takahashi S, Hori M, Tsuda K, Nakamura H. Diffusion-weighted single-shot echoplanar MR imaging for liver disease. AJR Am J Roentgenol 1999;173:393-398.

[4] Thoeny H, Ross B. Predicting and monitoring cancer treatment response with diffusion-weighted MRI. J Mag Res Imag 2010;32(1):2-16.

[5] Sandrasegaran K, Akisik F, Lin C, Tahir B, Rajan J, Saxena R, et al. Value of diffusion-weighted MRI for assessing liver fibrosis and cirrhosis. AJR Am J Roentgenol 2009;193(6):1556-1560.

[6] Jha A, Rodríguez J. A maximum-likelihood approach for ADC estimation of lesions in visceral organs. In: Image Analysis and Interpretation (SSIAI), 2012 IEEE Southwest Symposium on. IEEE; 2012, p. 21-24.

[7] Aja-Fernández S, de Luis Garcia R, Alberola-Lopez C, Hernando D. Quantitative diffusion MRI in the presence of noise: Effects of filtering and fitting technique. In: Quantitative Medical Imaging. Optical Society of America; 2013, p. QTu2G-2. 
[8] Abhinav J, Rodríguez J, Stopeck A. A maximum-likelihood method to estimate a single ADC value of lesions using diffusion MRI. Magn Reson Med 2016;76(6):1919-1931.

[9] Stephen R, Jha A, Roe D, Trouard T, Galons J, Kupinski M, et al. Diffusion MRI with semi-automated segmentation can serve as a restricted predictive biomarker of the therapeutic response of liver metastasis. Magn Reson Imaging 2015;33(10):1267-1273.

[10] Theilmann R, Borders R, Trouard T, Xia G, Outwater E, RangerMoore J, et al. Changes in water mobility measured by diffusion MRI predict response of metastatic breast cancer to chemotherapy. Neoplasia 2004;6:831-837.

[11] McLeish K, Hill D, Atkinson D, Blackall J, Razavi R. A study of the motion and deformation of the heart due to respiration. IEEE Trans Med Imag 2002;21(9):1142-1150.

[12] Zaitsev M, Maclaren J, Herbst M. Motion artifacts in MRI: A complex problem with many partial solutions. J Magn Reson Imaging 2015;42(4):887-901.

[13] Wachinger C, Navab N. Simultaneous registration of multiple images: Similarity metrics and efficient optimization. IEEE Trans Pattern Anal Mach Intell 2013;35(5):1221-1233.

[14] Kurugol S, Freiman M, Afacan O, Domachevsky L, Perez-Rossello J, Callahan M, et al. Motion-robust parameter estimation in abdominal diffusion-weighted mri by simultaneous image registration and model estimation. Med Imag Anal 2017;39:124-132.

[15] Taimouri V, Afacan O, Perez-Rossello J, Callahan M, Mulkern R, Warfield S, et al. Spatially constrained incoherent motion method improves diffusion-weighted mri signal decay analysis in the liver and spleen. Med Phys 2015;42(4):1895-1903.

[16] Kornapoulos E, Zacharaki E, Zerbib P, Lin C, Rahmouni A, Paragios N. Deformable groupwise registration using a physiological model: Application to diffusion-weighted mri. In: IEEE International Conference on Image Processing (ICIP). Arizona, United States; 2016, p. 2345-2349.

[17] Sanz-Estébanez S, Rabanillo-Viloria I, Royuela-del-Val J, Aja-Fernández $\mathrm{S}$, Alberola-López C. Joint groupwise registration and ADC estimation in the liver using a b-value weighted metric. Magn Reson Imaging 2018;46:1-9.

[18] Dikaios N, Punwani S, Hamy V, Purpura P, Rice S, Forster M, et al. Noise estimation from averaged diffusion weighted images: Can unbiased quantitative decay parameters assist cancer evaluation? Magn Reson Med 2014;71(6):2105-2117. 
[19] Walker-Samuel S, Orton M, McPhail L, Robinson S. Robust estimation of the apparent diffusion coefficient (ADC) in heterogeneous solid tumors. Magn Reson Med 2009;62:420-429.

[20] Weisskoff R, Baker J, Belliveau J, Davis T, Kwong K, Cohen M, et al. Power spectrum analysis of functionally-weighted MR data: What's in the noise. In: Proc Soc Magn Reson Med; vol. 1. 1993, p. 7.

[21] Aja-Fernández S, Vegas-Sánchez-Ferrero G. Statistical Analysis of Noise in MRI. Springer; 2016.

[22] Sijbers J, den Dekker A, Scheunders P, Van Dyck D. Maximumlikelihood estimation of Rician distribution parameters. IEEE Trans Med Imag 1998;17:357-361.

[23] Pruessmann K, Weiger M, Scheidegger M, Boesiger P. SENSE: sensitivity encoding for fast MRI. Magn Reson Med 1999;42(5):952-962.

[24] Thunberg P, Zetterberg P. Noise distribution in SENSE- and GRAPPAreconstructed images: a computer simulation study. Magn Reson Imaging 2007;25:1089-1094.

[25] Landman B, Bazin PL, Prince J. Diffusion tensor estimation by maximizing Rician likelihood. In: Computer Vision, 2007. ICCV 2007. IEEE 11th International Conference on. IEEE; 2007, p. 1-8.

[26] Aja-Fernández S, Alberola-López C, Westin C. Noise and signal estimation in magnitude MRI and Rician distributed images: a LMMSE approach. IEEE Trans Image Process 2008;17(8):1383-1398.

[27] Aja-Fernández S, Niethammer M, Kubicki M, Shenton M, Westin C. Restoration of DWI data using a Rician LMMSE estimator. IEEE Trans Med Imag 2008;27(10):1389-1403.

[28] Manjón JV, Coupé P, Concha L, Buades A, Collins DL, Robles M. Diffusion weighted image denoising using overcomplete local PCA. PloS one 2013;8(9):e73021.

[29] Tristán-Vega A, García-Pérez V, Aja-Fernández S, Westin C. Efficient and robust non-local means denoising of MR data based on salient features matching. Comput Methods Programs Biomed 2012;105:131-144.

[30] Reischauer C, Gutzeit A. Image denoising substantially improves accuracy and precision of intravoxel incoherent motion parameter estimates. PloS one 2017;12(4):e0175106.

[31] Le Bihan D, Breton E, Lallemand D, Aubin M, Vignaud J, Laval-Jeantet M. Separation of diffusion and perfusion in intravoxel incoherent motion MR imaging. Radiology 1988;168(2):497-505. 
[32] Kurugol S, Freiman M, Afacan O, Domachevsky L, Perez-Rossello J, Callahan M, et al. Motion compensated abdominal diffusion weighted MRI by simultaneous image registration and model estimation (SIR-ME). Med Imag Anal 2017;17:64-6.

[33] Le Bihan D, Breton E. Imagerie de diffusion in-vivo par résonance magnétique nucléaire. Comptes-Rendus de l'Académie des Sciences 1985;301(15):1109-1112.

[34] Stejskal E, Tanner J. Spin diffusion measurements: Spin echoes in the presence of a time-dependent field gradient. J Chem Phys 1965;42(1):288.

[35] Rueckert D, Aljabar P, Heckemann R, Hajnal J, Hammers A. Diffeomorphic registration using b-splines. MICCAI 2006 Lecture Notes in Computer Science 2006;4191:702-709.

[36] Veraart J, Sijbers J, Sunaert S, Leemans A, Jeurissen B. Weighted linear least squares estimation of diffusion MRI parameters: Strengths, limitations, and pitfalls. NeuroImage 2013;81:335-346.

[37] Pięciak T, Aja-Fernández S, Vegas-Sánchez-Ferrero G. Non-stationary Rician noise estimation in parallel MRI using a single image: a variance-stabilizing approach. IEEE Trans Pattern Anal Mach Intell 2017;39(10):2015-2029.

[38] Aja-Fernández S, Pięciak T, Vegas-Sánchez-Ferrero G. Spatially variant noise estimation in MRI: An homomorphic approach. Med Image Anal $2015 ; 20: 184-197$.

[39] Bernstein M, King K, Zhou X. Handbook of MRI Pulse Sequences. Burlington, MA: Elsevier Academic Press; 2004.

[40] Fieremans E. Validation methods for diffusion weighted magnetic resonance imaging in brain white matter. Ph.D. thesis; Doctoral dissertation, Ghent University; 2008.

[41] Du H, Wang L, Liu W, Yang F, Li Z, Zhu Y. Diffusion MRI simulation for human brain based on the atlas. In: 13th IEEE ISBI. Prague, Czech Republic; 2016, p. 898-902.

[42] Heidi J, Timothy E. Diffusion MRI. 1st ed. New York: Elsevier; 2009.

${ }_{625}$ [43] Luna A, Ribes R, Soto J. Diffusion MRI outside the Brain. SpringerVerlag; 2012.

[44] Segars W, Sturgeon G, Mendonca S, Grimes J, Tsui B. 4D XCAT phantom for multimodality imaging research. Med Phys 2010;37:49024915. 
[45] Sanz-Estébanez S, Peña-Nogales O, de-Luis-García R, Aja-Fernández S, Alberola-López C. Groupwise non-rigid registration on multiparametric abdominal DWI acquisitions for robust ADC estimation: Comparison with pairwise approaches and different multimodal metrics. In: 14th IEEE ISBI. Melbourne, Australia; 2017, p. 1156-1159.

635

[46] Learned-Miller E. Data driven image models through continuous joint alignment. IEEE Trans Pattern Anal Mach Intell 2006;28:236-250.

[47] Heinrich M, Jenkinson M, Bhushnan M, Matin T, Gleeson F, Brady $\mathrm{M}$, et al. Mind: Modality independent neighbourhood descriptor for multimodal deformable registration. Med Image Anal 2012;16:1423-1435.

[48] Pennec X, Cachier P, Ayache N. Non-local understanding the demons algorithm: 3D non-rigid registration by gradient descentshape descriptor: A new similarity metric for deformable multi-modal registration. MICCAI 1999 Lecture Notes in Computer Science 1999;1679:597-606.

[49] Aja-Fernández S, Krissian K. An unbiased non-local means scheme for DWI filtering. In: MICCAI: Workshop on Computational Diffusion MRI. Lecture Notes in Computer Science. Springer; 2008, p. 277-284.

[50] Buades A, Coll B, Morell J. A non local algorithm for image denoising. In: IEEE Computer Society CVPR 2005. San Diego, USA; 2005, p. 60-65.

[51] Maggioni M, Katkovnik V, Egiazarian K, Foi A. A nonlocal transformdomain filter for volumetric data denoising and reconstruction. IEEE Trans Image Process 2013;22(1):119-133. 